\title{
Tradeoffs between safe/ comfortable headways versus mobility-enhancing headways in an automated driving environment: preliminary insights using a driving simulator experiment
}

Comfortable vs mobilityenhancing headways

\author{
Yujie Li \\ Purdue University, West Lafayette, Indiana, USA \\ Tiantian Chen \\ Industrial and Systems Engineering, The Hong Kong Polytechnic University, \\ Kowloon, China, and \\ Sikai Chen and Samuel Labi \\ Purdue University, West Lafayette, Indiana, USA
}

\begin{abstract}
Purpose - The anticipated benefits of connected and autonomous vehicles (CAVs) include safety and mobility enhancement. Small headways between successive vehicles, on one hand, can cause increased capacity and throughput and thereby improve overall mobility. On the other hand, small headways can cause vehicle occupant discomfort and unsafety. Therefore, in a CAV environment, it is important to determine appropriate headways that offer a good balance between mobility and user safety/comfort.

Design/methodology/approach - In addressing this research question, this study carried out a pilot experiment using a driving simulator equipped with a Level-3 automated driving system, to measure the threshold headways. The Method of Constant Stimuli (MCS) procedure was modified to enable the estimation of two comfort thresholds. The participants (drivers) were placed in three categories ("Cautious," "Neutral" and "Confident") and 250 driving tests were carried out for each category. Probit analysis was then used to estimate the threshold headways that differentiate drivers' discomfort and their intention to re-engage the driving tasks. Findings - The results indicate that "Cautious" drivers tend to be more sensitive to the decrease in headways, and therefore exhibit greater propensity to deactivate the automated driving mode under a longer headway relative to other driver groups. Also, there seems to exist no driver discomfort when the CAV maintains headway up to $5 \%-9 \%$ shorter than the headways they typically adopt. Further reduction in headways tends to cause discomfort to drivers and trigger take over control maneuver.

Research limitations/implications - In future studies, the number of observations could be increased further. Practical implications - The study findings can help guide specification of user-friendly headways specified in the algorithms used for CAV control, by vehicle manufacturers and technology companies. By measuring and learning from a human driver's perception, AV manufacturers can produce personalized AVs

(C) Yujie Li, Tiantian Chen, Sikai Chen and Samuel Labi. Published in Frontiers in Engineering and Built Environment. Published by Emerald Publishing Limited. This article is published under the Creative Commons Attribution (CC BY 4.0) licence. Anyone may reproduce, distribute, translate and create derivative works of this article (for both commercial and non-commercial purposes), subject to full attribution to the original publication and authors. The full terms of this licence may be seen at http:// creativecommons.org/licences/by/4.0/legalcode

This work was supported by Purdue University's Center for Connected and Automated Transportation (CCAT), a part of the larger CCAT consortium, a USDOT Region 5 University Transportation Center funded by the U.S.DOT Award \#69A3551747105. The contents of this paper reflect the views of the authors, who are responsible for the facts and the accuracy of the data presented herein, and do not necessarily reflect the official views or policies of the sponsoring organization.
\end{abstract}

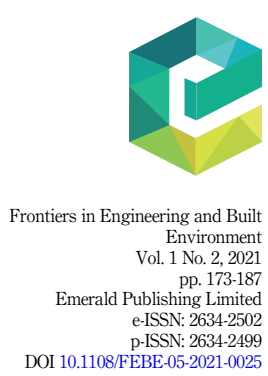


FEBE 1,2

\section{4}

Figure 1.

Conceptual tradeoff between travel efficiency and driver comfort to suit the user's preferences regarding headway. Also, the identified headway thresholds could be applied by practitioners and researchers to update highway lane capacities and passenger-car-equivalents in the autonomous mobility era.

Originality/value - The study represents a pioneering effort and preliminary pilot driving simulator experiment to assess the tradeoffs between comfortable headways versus mobility-enhancing headways in an automated driving environment.

Keywords Autonomous vehicles, Automated driving system, Connected vehicles, Driver perception, Car-following headway

Paper type Research paper

\section{Introduction}

Connected and autonomous vehicle (CAV) technologies provide promising solutions to transportation challenges that have plagued transportation systems for decades. Past research findings suggest that CAV adoption can contribute to the enhancement of safety, productivity and capacity of existing highway transportation corridors (Ha et al., 2020a; Du et al., 2020; Dong et al., 2021). There is a growing body of research that document the impacts of deploying the V2X communication (including vehicle-to-vehicle (V2V) and vehicle-toinfrastructure (V2I)) to facilitate roadway environment monitoring, trajectory planning and decision-making in automated driving mode (Darbha et al., 2018; Jung et al., 2020; Dong et al., 2020a, b; Chen et al., 2020, 2021a). For example, with V2X communication, CAVs are afforded not only a superior level of awareness of their surrounding environments (Chen, 2019) but also opportunity to form platoons that are associated with smaller headways (Li et al., 2020a, b; Ha et al., 2020b). Such formation of platoons is governed by the AI-based control algorithms in CAVs. When the headways are too large, the throughput is reduced and travel efficiency suffers. On the other hand, when the headways are too small, drivers may feel unsafe and anxious, and therefore deactivate the automated driving system (ADS) by taking over the vehicle control from the ADS. The research question, therefore, is: to what extent can headways can be reduced so that there is a good balance between safe and comfortable headways versus mobility-enhancing headways (see Figure 1).

In the literature, the two common expressions of headway are distance headway and time headway. Distance headway refers to the bumper-to-bumper distance between the lead vehicle and the following vehicle, while time headway refers to the time interval between two vehicles in car-following scenarios (Fuller, 1981; Winsum et al., 1996). As an important safety indicator, headway represents a measure of the potential danger associated with a traffic situation, and is commonly used by authorities for enforcement purposes (Vogel, 2003). For example, Hong Kong, France and Netherlands recommend a safe time headway of $2 \mathrm{~s}$, while a lower safetycritical threshold of $1 \mathrm{~s}$ is adopted in Sweden (Risto and Martens, 2013; Vogel, 2003). With regard to the distance headway, drivers tend to gage the distance to the vehicles they follow based on their perception of the physical distance (Cutting and Vishton, 1995). Compared with time

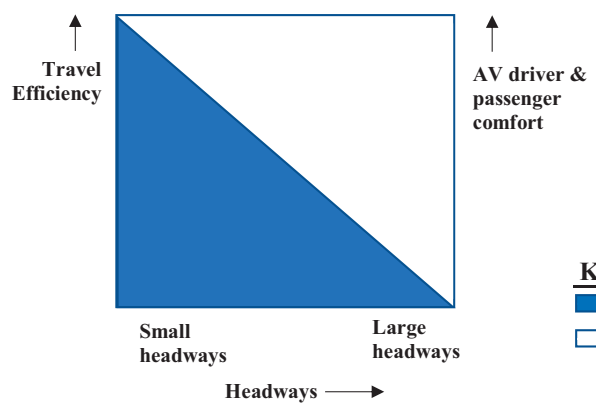


headway, driver's judgment in distance headway could be influenced to a greater extent by the variations in vehicle speed and the target physical distance (Risto and Martens, 2013). Also, the safe headway distance is lower when traffic density is high. Abuelenin and Abul-Magd (2015) proposed that the minimum safety distance headway should decrease from 40 to 10 meters when the density increases from 25 to $40 \mathrm{veh} / \mathrm{km}$.

Furthermore, previous research has indicated that human drivers' preferred headways vary across different traffic conditions (Taieb-Maimon and Shinar, 2001; Suzuki and Nakatsuji, 2015). Indeed, drivers' risk perception influences driving behavior. For example, previous studies showed that older drivers perceive higher safety risk and tend to keep longer headways (Andrews and Westerman, 2012; Charlton et al., 2006; Shinar et al., 2005; Ni et al., 2010; Martchouk et al., 2010). Chen et al. (2021a) found that under the high-volume traffic conditions, experienced drivers tend to adopt longer time-headways in order to reduce crash risk. Also, past research has established that driver behaviors and perceptions vary across different vehicle classes and types including bus, taxi, trucks and private cars (Chen et al., 2021b). Also, when the headway gets smaller, drivers perceive higher rear-end collision risk and report increased levels of unsafety and discomfort (Siebert et al., 2014; Lewis-Evans et al., 2010). In particular, Lewis-Evans et al. (2010) investigated the impact of time headways ranging from 0.5 to $4.0 \mathrm{~s}$ (pre-determined by the Adaptive Cruise Control (ACC) system). The authors found that drivers' subjective ratings of safety risk and discomfort level increased significantly when headways reach a threshold value of $2 \mathrm{~s}$. In a subsequent experiment, Siebert et al. (2014) determined the time-headway thresholds at different speeds (50, 100, $150 \mathrm{~km} / \mathrm{h}$ ). It is critical to note that, there is a gap between the comfortable distance headway and the minimum safe distance headway adopted by drivers (Duan et al., 2013). The authors found that when they were instructed to "keep a comfortable distance," drivers tend to adopt a longer headway compared with when they were instructed to "keep a minimal safe distance." Piccinini et al. (2014) also found that even with vehicles equipped with an ACC, drivers still tend to adopt longer headways compared to the group tested with driving without ACC. The findings of the aforementioned earlier studies not only threw more light on driver headway propensities but also accentuated the need for enhanced understanding of driver headway requirements and preferences particularly in the emerging age of vehicle automation.

In the literature, it is clear that drivers' headway preferences under different driving conditions have been examined to some extent. Yet still, there is rather limited research on the difference in driver's comfort level between the vehicle-following distance decided by human drivers and that decided by the automated driving system. This is an important research question with profound practical consequences because it is anticipated that in the foreseeable future, the traffic stream will be characterized by a mix of human driven vehicles and CAVs. Previous studies showed that drivers tend to deactivate the automated driving mode when the they have low trust in automation (Deo and Trivedi, 2019; Hengstler et al., 2016; Molnar et al., 2018; Miller et al., 2016; Petersen et al., 2019). Nevertheless, the bigger picture seems to be associated with sanguine outcomes: smaller time headways will lead to increased lane capacity and reduced delay. Therefore, it is critical to identify the headway threshold by analyzing the trade-offs between the user-friendly headways (to ensure drivers' comfort level and safety) and smaller headways (to enhance overall mobility) in an automated driving environment.

In the emerging age of vehicle automation, it is anticipated that the role of humans as drivers will shift to that of passengers - this phenomenon has been termed "the loss of controllability" (Elbanhawi et al., 2015). Past studies that used driving simulation suggest that drivers of Level 3 automated driving tend to take over control of the vehicle's automated driving system based on their judgments on road traffic conditions (Forster et al., 2017; Merat et al., 2014; Wright et al., 2018). This suggests that, first, some level of mistrust that human 
FEBE

1,2

drivers harbor towards automated driving systems, and second, these drivers perceive the ADS only as a backup driving system (Payre et al., 2016). In the extreme cases of Levels 4 or 5 automation where human control of the driving task is severely limited or non-existent, respectively, the lack of take over opportunity may lead to driver/passenger anxiety. As such, it is essential to assess the driver needs regarding headways comfortability in the context of AVs.

To address this issue, it is possible to carry out driving simulator experiments where driver perception and behaviors can be observed in a controlled environment. Compared to other test platforms including test tracks and in-service roads, driving simulation represents a safe, cost-effective and flexible platform for driving behavior investigation (Boyle and Lee, 2010; Chen et al., 2019a, b, 2021a; Fisher et al., 2011). The present study prospectively contributes to the literature by using driving simulator experiments to carry out headway threshold design in the context of CAV environment considering driver's discomfort levels and their takeover intention, and to analyze the tradeoffs between safe/comfortable headways versus mobility-enhancing headways in the automated driving environment.

The driving simulator in the present study is equipped with level 3 automated driving system (ADS) that specified in the SAE standard (SAE International, 2018). In particular, a level $3 \mathrm{ADS}$ feature requires drivers to be vigilant and ready to take over the vehicle under some safety-critical situations. Also, it is important that the level 3 ADS-equipped vehicles are able to adopt proper headways. This is because shorter headway without the careful consideration of driver perception will lead to unnecessary takeovers. Drivers are expected to re-engage in driving tasks as they feel uncomfortable or perceive any close car-following as unsafe. In addition, the Method of Constant Stimuli is used, with some modification, to measure the quantitative relationship between the stimulus (different levels of distance headway) and driver perception (Gescheider, 1985; Simpson, 1988; Leek, 2001).

The remainder of this paper is structured as follows. Section 2 provides details of experimental design, procedures of driving simulator test and the method of analysis. Sections 3 and 4 presents the results and discussions, respectively. Section 5 concludes the paper with a summary of the findings and future research directions.

\section{Methods}

\subsection{Participants}

Drivers recruited for the driving simulator experiment are male college students at Purdue University. The selection criteria were: the possession of a valid full driving license, having driving experience in the United States, and (self-declared) good health condition. In spite of the best efforts of the researchers, no female driver was available for experiment at the time of the study. This situation was considered not debilitating to the study, due to the purpose of the study as an initial and preliminary pilot experiment. Female drivers are being recruited by the researchers in a follow-up study. Participation in the experiment was voluntary and informed consent was obtained from each participant. An initial screening was performed prior to the experiment. All the participants were fully conscious prior to the start of experiment since the current study intended to collect the participants' (drivers') perception during the simulation task. Therefore, the participants were required to have a good rest on the day before the experiment and abstained from consumption of alcohol and caffeinated beverages $24 \mathrm{~h}$ before the experiment. The participants were placed into three categories based on their stated level of caution in driving. Drivers in group 1 stated a high confidence in driving task (i.e. lower level of cautious driving), drivers of group 3 stated a low confidence in driving task (i.e. higher level of cautious driving), while drivers of group two reported a middle level. Therefore, groups 1, 2 and 3 were further labeled as "confident," "neutral" and "cautious." 


\subsection{Equipment and driving scenarios}

The driving simulator experiments were conducted on a fixed-base device simulator manufactured by AVSimulation (Figure 2). The simulator is equipped with three full HD-LED displays, steering wheel (with force-feedback), pedals (clutch, brake, and accelerator), dashboard (speedometer, control buttons), headlight and turn-signal, mode-shift function (automated or manual mode) and sound system. SCANeR ${ }^{\mathrm{TM}}$ studio software was used to generate the simulated driving scenario, where high-resolution images were created to simulate the scenery of the road environment along a particular highway section in Indiana a straight road segment on Interstate 465 in northern Indianapolis (Figure 3). A key operational capability of the simulator is the ability to switch between autonomous and manual modes. This transition function enables the participant to change to manual mode in situations where the participant feels uncomfortable or unsafe anytime during the automated driving system's operations. In this study, subjective responses to indicate driver's discomfort levels were measured using a questionnaire.

\subsection{Test procedures}

2.3.1 Training session. A practice run was provided to help the drivers familiarize themselves with the simulator control (particularly, activation of the automated driving mode and the
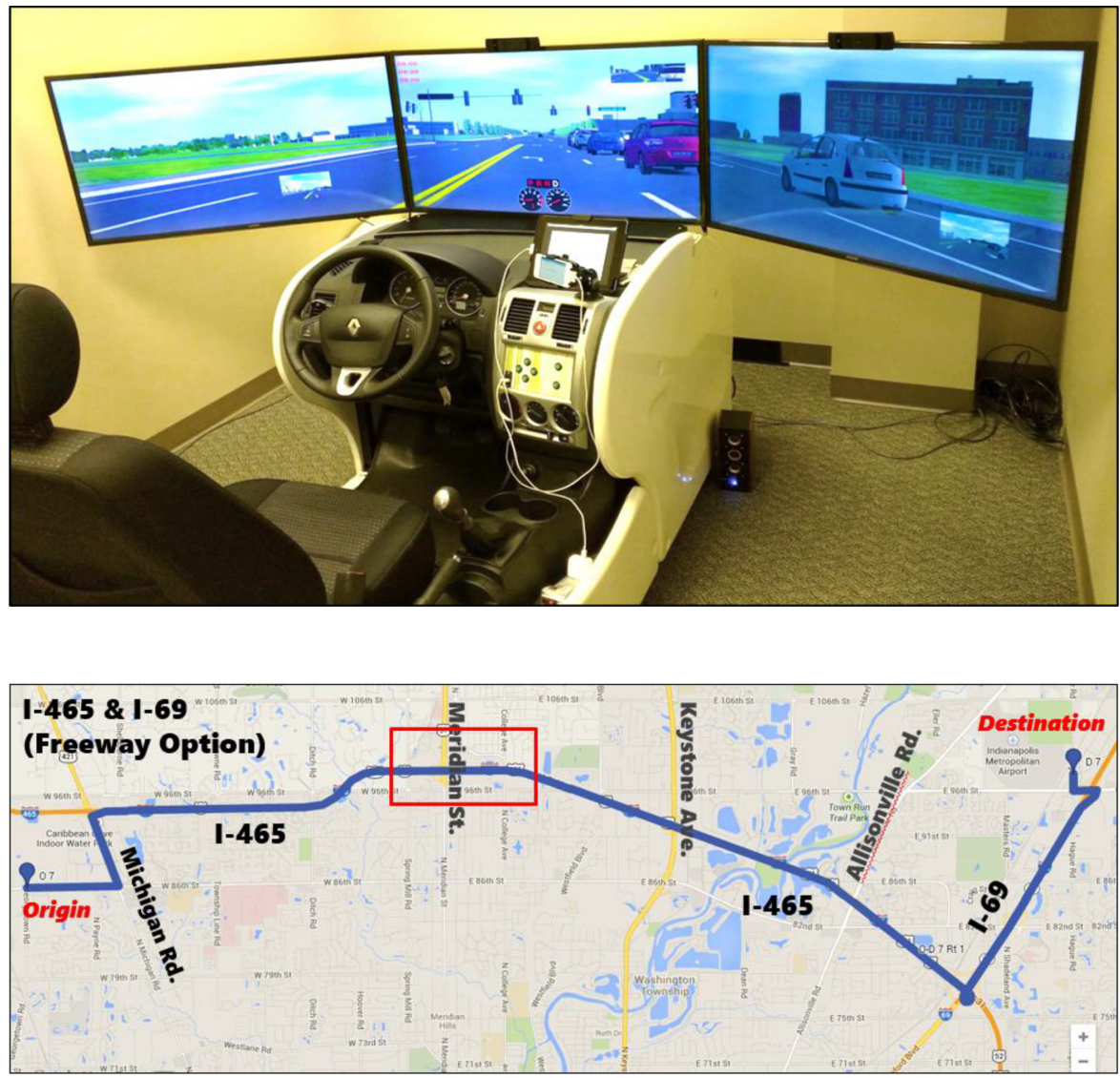

Comfortable vs mobilityenhancing headways

Figure 2 .

Cockpit of the driving simulator used in the experiment
Figure 3.

Test section: straightline I-465 segment in Indianapolis (see boxed segment) 
FEBE 1,2

take-over functions) and to identify individuals that may have simulator sickness. As the simulator is equipped with Level 3 automated driving, drivers were informed of their responsibility to monitor the system performance and to take over (switch to manual mode) if and when they felt it was necessary to do so. The participants were instructed as follows:

Because of the limitations of the enabling technologies that autonomous vehicles rely on, bad weather such as rain, snow, or fog can interfere with the signals from the various sensors. Also, poor lane markings or construction zones can cause the automation to disengage. When the vehicle enters conditions it cannot handle, it will issue a request for the driver to resume vehicle control. The level 3 automated driving system can initiate a take-over request at any time during the drive. It is the responsibility of the driver to remain vigilant of traffic conditions and maintain your ability to resume the driving task when the automation signals that it has reached its limitations. Always remember that you are ultimately responsible for your vehicle and road safety at all times.

2.3.2 Manual driving session. Each participant was then asked to complete ten tests in manual mode. During each driving test, they were instructed to make manual control adjustments to ensure a comfortable following distance with a leading vehicle, and to switch the mode from manual driving to automated driving when they considered such "comfortable" level had been reached. Data related to the comfortable distance headways indicated by the drivers, were collected at the end of each trial. After completion of the ten tests (trials), the mean value $(\mu)$ and standard deviation $(\sigma)$ of the headways were calculated. Then, the 5 headway levels tested in the automated driving session were set to $\mu-2 \sigma, \mu-\sigma, \mu, \mu+\sigma, \mu+2 \sigma$.

2.3.3 Automated driving session. For the simulated driving tests under the automated driving mode, the vehicle was set to follow the leading vehicle with a predefined headway. Specifically, five levels of distance headways were chosen as the "stimuli" based on the reference values (i.e. the means and standard deviations of the distance headways obtained in Section 2.3.2). For each distance headway, 50 tests were conducted, yielding a total of 250 observations. The stimuli presented were randomized and counterbalanced across the participants. At the end of each test, participants were required to answer yes or no to the following questions:

\section{Q1. Did you experience any discomfort? Y/N \\ Q2. Did you want to take over the vehicle? $\mathrm{Y} / \mathrm{N}$}

The second question was asked only if the answer to the first question was "Yes". Figure 4 presents the procedure for measuring the headway thresholds. The drivers' discomfort levels were collected rather than their comfort levels, for two reasons. First, our pilot study showed that drivers tend to be more sensitive to discomfort compared to comfort. Secondly, one of the benefits of CAVs is that they are expected to have shorter headways compared to the conventional (human-driven) vehicles. As we hypothesized that drivers tend to feel more uncomfortable with decreases in distance headways, it would be more straightforward to measure the discomfort levels. Three levels of discomfort were further defined: "No discomfort," "Somewhat Uncomfortable" and "Very uncomfortable." If the answer to Q1 is "No," the presented headway was noted as "No Discomfort." If the answer is "Yes" and the answer to Q2 was "No," the presented headway was noted as "Somewhat Uncomfortable," and if the answer to Q2 was "Yes," the presented headway was noted as "Very Uncomfortable." Using the schema shown in Figure 5, this study developed a relationship between the stimulus (distance headways) and driver's discomfort level.

\subsection{Data analysis}

The objective of this experiment was to estimate two absolute thresholds (Figure 5) corresponding to different levels of discomfort. The first threshold distinguishes the "Very Uncomfortable" headway from the "Somewhat uncomfortable" headway; and the second 


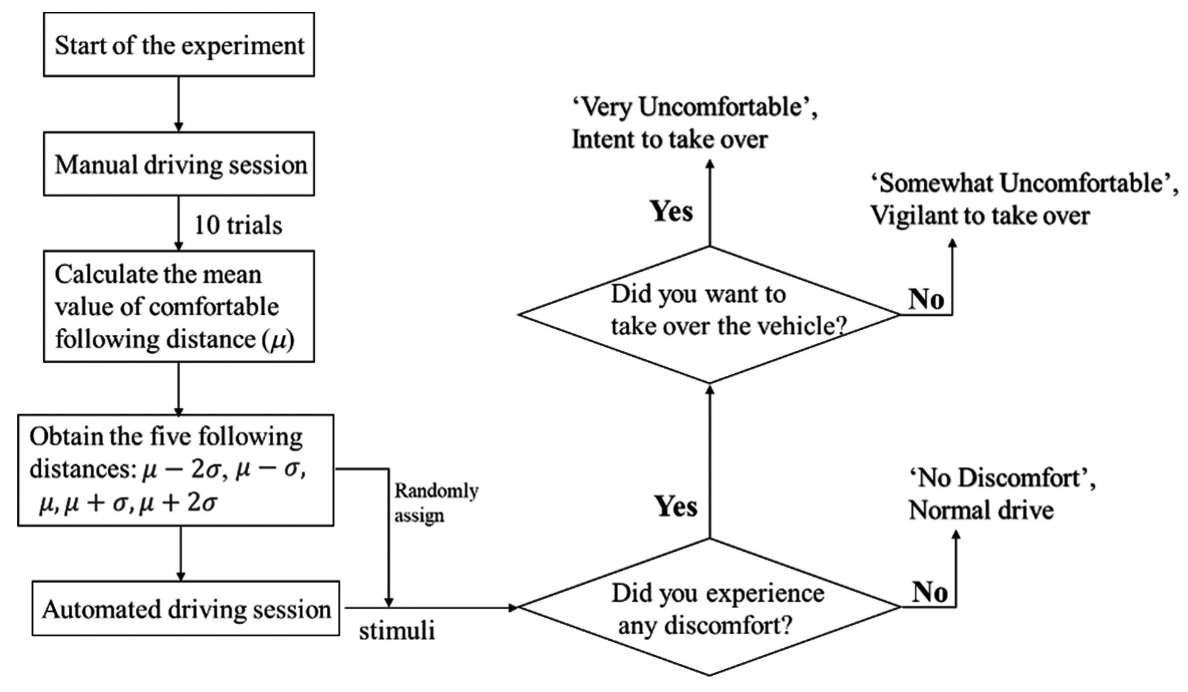

Comfortable vs mobilityenhancing headways

179

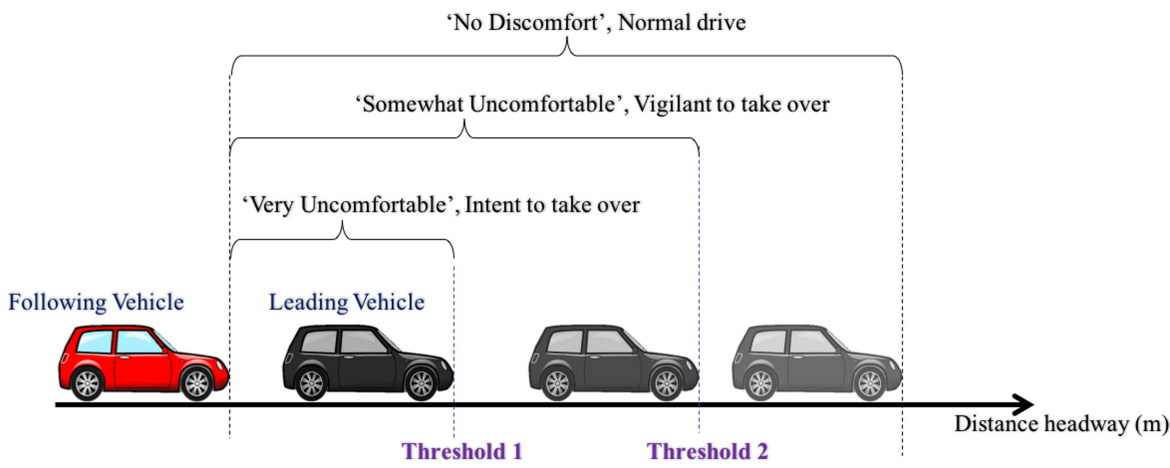

Figure 5.

Relationship between headways and level of discomfort

threshold distinguishes the "Somewhat Uncomfortable" from the "No Discomfort" headways. This study simultaneously measured the two thresholds by revising the Method of Constant Stimuli (Patten et al., 2006; Cantin et al., 2009). In the classic Method of Constant Stimuli, the proportion of detected responses are recorded and plotted as a function of stimulus intensity levels. Figure 6 presents an example of the psychometric graph. As a rule of thumb, when the intensity of the stimulus is extremely low, the probability of subjects reporting a stimulus detection is close to zero; on the other hand, when the intensity is high, subjects tend to confirm the detection of stimulus. When plotted with an adequate number of measurements, psychometric curves often follow a particular "S" shape termed "ogive" and a theoretical curve can be fitted to the observations. For this purpose, the cumulative Gaussian distribution is typically used for the curve fitting, and its efficacy in this regard is supported by theory as well as experimental findings in past research. Examples include the outcomes of psychometric studies that are found in biological and psychological science publications (Gescheider, 1985). The maximum likelihood technique can then be used to estimate the parameters (mean and standard variation) that characterize the Gaussian distribution. 
FEBE

1,2

180

Figure 6.

A typical psychometric graph obtained when the absolute threshold is measured

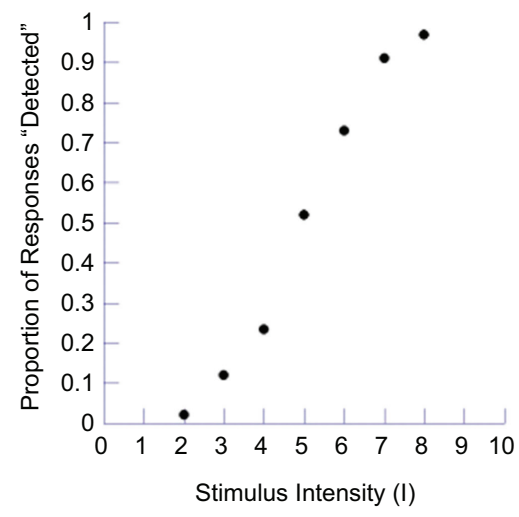

(a)

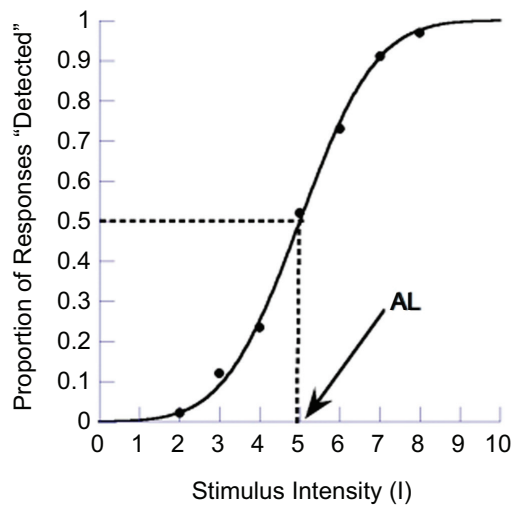

(b)

Figure 6(b) presents a curve drawn to fit the hypothetical points (observations) shown in Figure 6(a).

Using the fitted curve, the threshold is estimated as the stimulus value corresponding to $50 \%$ detection (Figure 6(b)). In this experiment, it is sought to estimate two thresholds for comfort levels, and therefore we modified the Method of Constant Stimuli, as follows: to measure the headway threshold between "No discomfort" and the "Somewhat Uncomfortable" levels (i.e. Threshold 2), all the answers of "Yes" to the first question (Q1) were first counted into the "Uncomfortable" group. Then, the procedure of conventional constant stimuli method was applied to the two classes - "No Discomfort" and "Uncomfortable." The same procedure was applied to estimate the Threshold 1. The proportions of responses for each stimulus value were recorded. Then we fitted the data points using the cumulative Gaussian distribution. The two parameters (mean and standard deviation) of the Gaussian distribution were estimated using the maximum likelihood method (Probit analysis). The absolute threshold using the mean value of the Gaussian distribution were estimated in this study.

\section{Results}

Table 1 presents the headway measurements in both manual and AV modes. The estimation results of thresholds using the Probit analysis are presented in Figures 7(a)-(c). Different

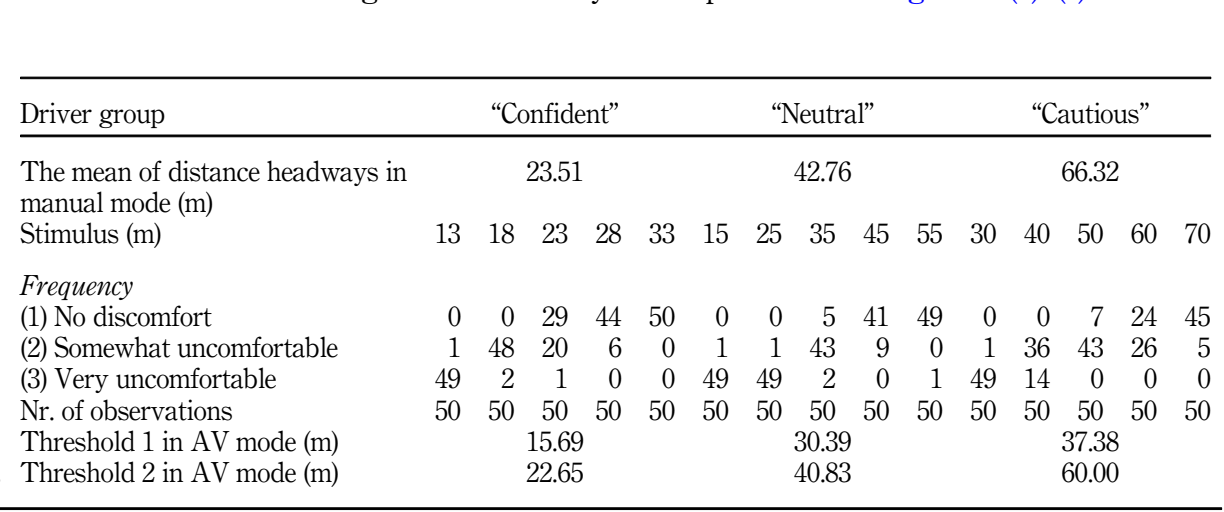

Table 1.

Headway measurements of the three groups of drivers 
thresholds were estimated for each driver group ("Confident," "Neutral" and "Cautious"). In particular, for the "Confident" driver group, when the distance headway is shorter that the estimated threshold value of 15.69 meters, drivers tend to take over the automated driving mode. Moreover, the "Confident" drivers tend to feel uncomfortable when the distance headway decreases to less than 22.65 meters. For the "Neutral" group, drivers tend to feel uncomfortable when the distance headway is shorter than 40.83 meters, and show their intention to take over the automated driving mode when the distance headway further reduces to less than 30.39 meters. For the last group - "Cautious" drivers, Threshold 1 and Threshold 2 were estimated as 60.0 meters and 37.38 meters, respectively.

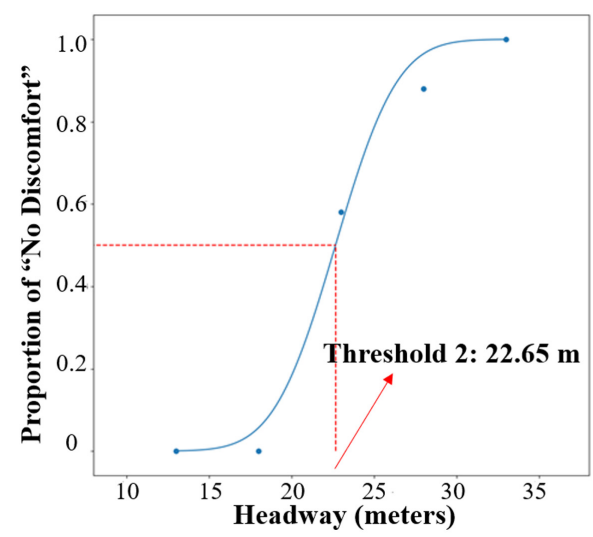

i. Estimation of Threshold 2

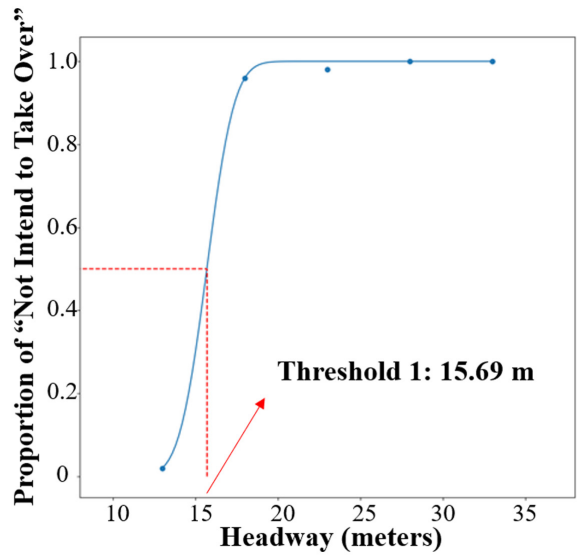

ii. Estimation of Threshold 1
Comfortable vs mobilityenhancing headways

'Confident' drivers

(a)

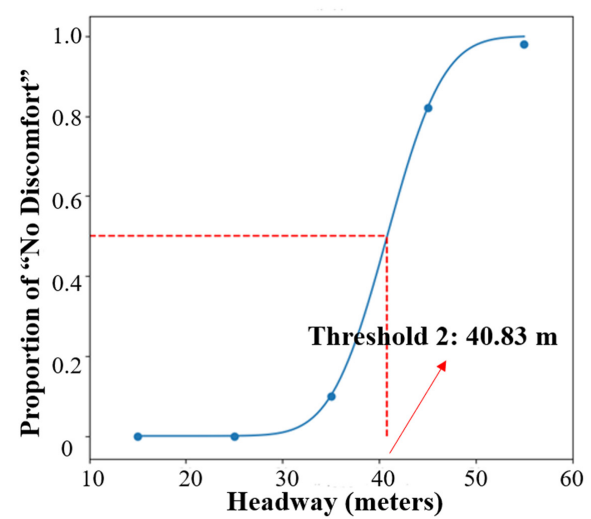

i. Estimation of Threshold 2

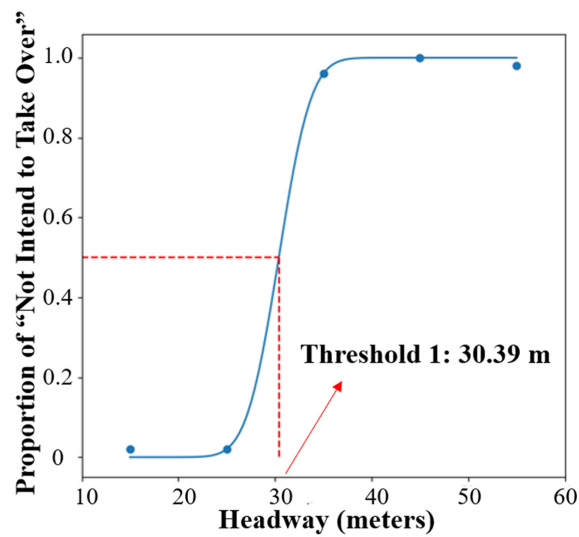

ii. Estimation of Threshold 1

'Neutral' drivers

Figure 7.

Determination of headway thresholds for various driver categories

(b) 


\section{FEBE} 1,2

182

Figure 7.

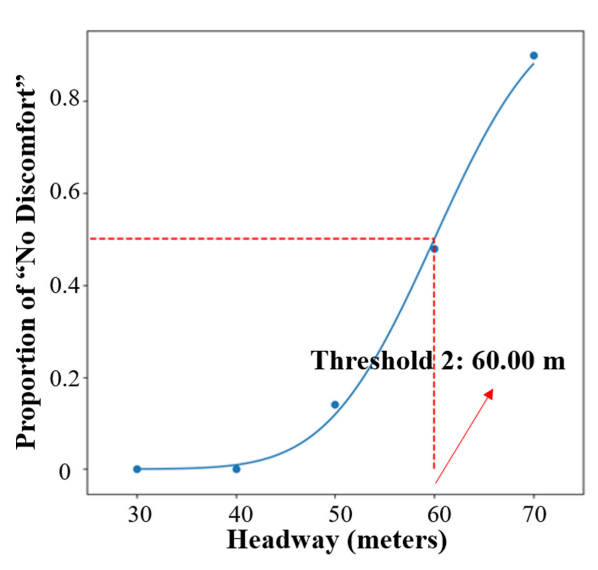

i. Estimation of Threshold 2

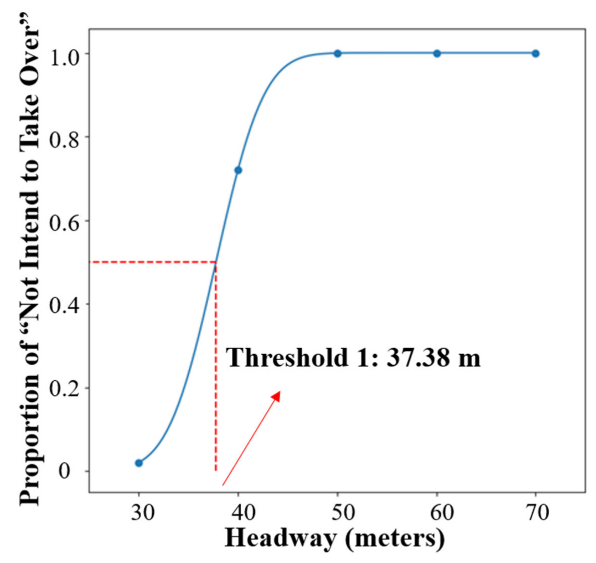

ii. Estimation of Threshold 1

'Cautious' drivers

(c)

\section{Discussion}

This study used driving simulator experiments to investigate drivers' comfortable carfollowing distances in a CAV environment. The driving simulator was equipped with level 3 automated driving. During the experiment, drivers were required to report their discomfort level and the intention to take over the vehicle, and this was used as a basis to measure the threshold headways. This is expected to enhance safety and CAV user comfort in the future where the traffic stream will contain a significant number of CAVs. In addition, the study results can help analysts determine the extent to which headway reduction, particularly for connected and automated buses or trucks in a platoon, can be expected to enhance overall mobility and productivity without jeopardizing occupant comfort. The study results also suggests that even in the far future where AVs are equipped with the highest level of automation (level 5 full automated driving system), the issues related to human factors should not be ignored, and that the safety and comfort of the occupant are of paramount importance. In particular, there exist great concern regarding the comfort level and trust that drivers and passengers associate with $\mathrm{AI}$ technologies, and this is a critical issue that needs to be addressed in CAV development and deployment. In this regard, the present research provides a significant contribution to furthering the understanding of the trade-offs in headways between the overall mobility and user experience in an AV environment.

In this study, different headway thresholds were estimated for the three groups of drivers operating at the same vehicle speed. The experiment outcomes suggest that "Cautious" drivers tend to be more sensitive to the decrease in headways, thus showing intention to deactivate automated driving mode under a longer headway relative to those for the other two groups. In addition, the results revealed that the reference headways measured under the manual driving mode are all slightly longer than the headway Threshold 2 under the automated driving mode. In other words, this suggests that drivers did not report discomfort even where the CAV maintained a headway shorter than the headways the drivers typically adopt.

These results could be explained by the previous findings that the intention to take over the automated driving mode is associated with the trust in AI technology (Deo and Trivedi, 2019; Hengstler et al, 2016; Miller et al, 2016; Petersen et al, 2019). Molnar et al. (2018) pointed out that the trust in automated driving and the acceptance of technology would influence the decision of 
transition between automated mode and manual mode. The authors found that one unit increase in trust level is associated with $15 \%$ more scenarios in which the driver choose to activate the automated driving mode. Moreover, Du et al. (2020) found that some physiological factors (reflecting drivers' workload and stress) can significantly affect the driver's takeover performance in Level 3 automated driving.

With regard to the effect of driver types, it has been shown in the literature (Chen et al. (2021) that older drivers tend to be more cautious and therefore tend to adopt longer headways. Comparatively, their mid-aged counterparts are more confident with their driving skills and therefore follow the leading vehicle with shorter headways. Underwood (2013) indicated that novice drivers tend to be cautious followers and maintain a longer headway with the leading vehicle. Irrespective of the effects of driver age and experience, it has been shown in previous studies that cautious driving style is correlated with significantly longer headway behavior (Bao et al., 2020; Ivanco, 2017; Saifuzzaman et al., 2015; Shinar and Schechtman, 2002). Consistent with these past findings, our study results suggest that individual driving style also significantly influence headway acceptance or comfort level in the context of CAV environment. In particular, the "Cautious," "Neutral" and "Confident" drivers tend to deactivate the automated driving mode when the distance headway reach a threshold of 37.38 meters, 30.39 meters, and 15.69 meters, respectively.

The findings from this research are expected to provide useful insights for AV manufacturers and technology companies regarding the CAV design, to help foster user acceptance (Vob et al., 2018). For example, by learning from the human driver's perception (e.g. different comfortable headways), studies of this nature can yield results that enable the car manufacturer to personalize the AV for a specific individual. Moreover, Martin-Gasulla et al. (2019) pointed out that the less cautious car-following behavior (time headway decrease from $1.8 \mathrm{~s}$ to $0.6 \mathrm{~s}$ ) of CAVs contributes to a $15 \%$ delay reduction. However, the results of this study suggest that capacities and delay should be updated using the "use-friendly" headways in CAVs-operation environment. Therefore, transportation planning involving autonomous mobility could be enhanced in the long run.

\section{Concluding remarks}

The simulator experiment in this study help measure the threshold headways to identify driver's discomfort and intention to deactivate the automated driving mode. This was done for each of three driver types (i.e. "Cautious," "Neutral" and "Confident"). In particular, an innovative constant stimuli method which can estimate two absolute thresholds in one session was adopted in this study. Five stimuli based on the reference headways obtained under the manual driving mode were used as the stimuli under the level 3 automated driving mode. Drivers' responses to two questions regarding discomfort levels and takeover intention were collected. The results reveal the longer headway thresholds of "Cautious" drivers (i.e. 60.0 meters to report discomfort; 37.38 meters to take over the vehicle) as compared with the "Confident" drivers (i.e. 22.65 meters to report discomfort; 15.69 meters to take over the vehicle). This could be attributed to the effects of physiological factors, driving style, trust in automated driving, and the acceptance of AI technology. The findings from this research are expected to provide useful insights into the balancing act between the enhanced mobility and user experience associated with vehicle headways in a CAV environment.

The study has a few limitations that could be addressed in future research. First, a driver's judgment in distance headway can be influenced by vehicle speed, traffic volume and road conditions. However, this study did not account for the effects of operational and environmental factors on drivers' comfortable following headways, and these could be considered in future work. Second, the drivers' discomfort levels could be measured using objective rather than subjective means, for example, using electrocardiogram (ECG) 
FEBE 1,2

\section{4}

equipment. Third, past exposure to automation or technology could be affecting drivers' trust in automated driving and subsequently their comfortable headways. However, information on their past exposure was not collected in the current study. Fourth, the recruited drivers in this study are all college students within the same age group. Also, future studies could include female participants in order to investigate whether headway comfort differs significantly across the two genders. In future research, it is essential to incorporate a wider and more diverse sample, to better understand how different socio-demographic characteristics are related to trust in automated driving systems. Last, in determining the headway of CAVs, future studies could consider AV-to-manual transition time to ensure that drivers have adequate time to take over the vehicle.

\section{References}

Abuelenin, S.M. and Abul-Magd, A.Y. (2015), "Effect of minimum headway distance on connectivity of VANETs", AEU-International Journal of Electronics and Communications, Vol. 69 No. 5, pp. 867-871.

Andrews, E.C. and Westerman, S.J. (2012), "Age differences in simulated driving performance: compensatory processes", Accident Analysis and Prevention, Vol. 45, pp. 660-668.

Bao, S., Wu, L., Yu, B. and Sayer, J.R. (2020), "An examination of teen drivers' car-following behavior under naturalistic driving conditions: with and without an advanced driving assistance system", Accident Analysis and Prevention, Vol. 147, 105762.

Boyle, L.N. and Lee, J.D. (2010), "Using driving simulators to assess driving safety", Accident Analysis and Prevention, Vol. 42 No. 3, pp. 785-787.

Cantin, V., Lavallière, M., Simoneau, M. and Teasdale, N. (2009), "Mental workload when driving in a simulator: effects of age and driving complexity", Accident Analysis and Prevention, Vol. 41 No. 4, pp. 763-771.

Charlton, J.L., Oxley, J., Fildes, B., Oxley, P., Newstead, S., Koppel, S. and O'Hare, M. (2006), "Characteristics of older drivers who adopt self-regulatory driving behaviours", Transportation Research Part F: Traffic Psychology and Behaviour, Vol. 9 No. 5, pp. 363-373.

Chen, S. (2019), Safety Implications of Roadway Design and Management: New Evidence and Insights in the Traditional and Emerging (Autonomous Vehicle) Operating Environments, Ph.D. Dissertation, Purdue University, West Lafayette.

Chen, T., Sze, N.N. and Bai, L. (2019a), "Safety of professional drivers in an ageing society-a driving simulator study", Transportation Res. Part F: Traffic Psychology and Behaviour, Vol. 67, pp. 101-112.

Chen, T., Bai, L. and Sze, N.N. (2019b), "Factors affecting the severity of rear-end conflicts: a driving simulator study", 2019 5th International Conference on Transportation Information and Safety, IEEE, pp. 1182-1187.

Chen, S., Leng, Y. and Labi, S. (2020), “A deep learning algorithm for simulating autonomous driving considering prior knowledge and temporal information", Computer-Aided Civil and Infrastructure Engineering, Vol. 35 No. 4, pp. 305-321.

Chen, S., Dong, J., Ha, P., Li, Y. and Labi, S. (2021a), "Graph neural network and reinforcement learning for multiagent cooperative control of connected autonomous vehicles", Computer-Aided Civil and Infrastructure Engineering, Vol. 36 No. 7, pp. 838-857.

Chen, T., Sze, N.N., Newnam, S. and Bai, L. (2021b), "Effectiveness of the compensatory strategy adopted by older drivers: difference between professional and non-professional drivers", Transportation Research Part F: Traffic Psychology and Behaviour, Vol. 77, pp. 168-180.

Chen, T., Sze, N.N., Chen, S., Labi, S. and Zeng, Q. (2021c), "Analysing the main and interaction effects of commercial vehicle mix and roadway attributes on crash rates using a Bayesian randomparameter Tobit model", Accident Analysis and Prevention, Vol. 154, 106089. 
Cutting, J.E. and Vishton, P.M. (1995), "Perceiving layout and knowing distances: the integration, relative potency, and contextual use of different information about depth", Perception of Space and Motion, Academic Press, pp. 69-117.

Darbha, S., Konduri, S. and Pagilla, P.R. (2018), "Benefits of V2V communication for autonomous and connected vehicles", IEEE Transactions on Intelligent Transportation Systems, Vol. 20 No. 5, pp. 1954-1963.

Deo, N. and Trivedi, M.M. (2019), "Looking at the driver/rider in autonomous vehicles to predict takeover readiness", IEEE Transactions on Intelligent Vehicles, Vol. 5 No. 1, pp. 41-52.

Comfortable vs mobilityenhancing headways

Dong, J., Chen, S., Li, Y., Du, R., Steinfeld, A. and Labi, S. (2021), "Space-weighted information fusion using deep reinforcement learning: the context of tactical control of lane-changing autonomous vehicles and connectivity range assessment”, Transportation Research Part C: Emerging Technologies, Vol. 128, 103192.

Dong, J., Chen, S., Li, Y., Ha, P.Y.J., Du, R., Steinfeld, A. and Labi, S. (2020a), "Spatio-weighted information fusion and DRL-based control for connected autonomous vehicles", 2020 IEEE 23rd International Conference on Intelligent Transportation Systems, pp. 1-6.

Dong, J., Chen, S., Ha, P., Du, R., Li, Y. and Labi, S. (2020b), "DRL-based multiagent cooperative control framework for CAV networks: a graphic convolution Q network", arXiv preprint arXiv: 2010.05437, available at: https://arxiv.org/abs/2010.05437.

Du, R., Chen, S., Li, Y., Dong, J., Ha, P. and Labi, S. (2020), “A cooperative control framework for CAV lane change in a mixed traffic environment", arXiv preprint arXiv:2010.05439, available at: https://arxiv.org/abs/2010.05439.

Duan, J., Li, Z. and Salvendy, G. (2013), "Risk illusions in car following: is a smaller headway always perceived as more dangerous?”, Safety Science, Vol. 53, pp. 25-33.

Elbanhawi, M., Simic, M. and Jazar, R. (2015), "In the passenger seat: investigating ride comfort measures in autonomous cars", IEEE Intelligent Transportation Systems Magazine, Vol. 7 No. 3, pp. 4-17.

Fisher, D.L., Rizzo, M., Caird, J.K. and Lee, J.D. (2011), Handbook of Driving Simulation for Engineering, Medicine, and Psychology. doi: 10.1201/b10836-2.

Forster, Y., Naujoks, F., Neukum, A. and Huestegge, L. (2017), "Driver compliance to take-over requests with different auditory outputs in conditional automation", Accident Analysis and Prevention, Vol. 109, pp. 18-28.

Fuller, R.G. (1981), "Determinants of time headway adopted by truck drivers", Ergonomics, Vol. 24 No. 6, pp. 463-474.

Gescheider, G.A. (1985), Psychophysics: Method, Theory, and Application, Psychology Press, Hillsdale.

Ha, P., Chen, S., Du, R., Dong, J., Li, Y. and Labi, S. (2020a), "Leveraging the capabilities of connected and autonomous vehicles and multi-agent reinforcement learning to mitigate highway bottleneck congestion", arXiv preprint arXiv:2010.05436, available at: https://arxiv.org/abs/ 2010.05436 .

Ha, P.Y.J., Chen, S., Du, R., Dong, J., Li, Y. and Labi, S. (2020b), "Vehicle connectivity and automation: a sibling relationship", Frontiers in Built Environment, Vol. 6, p. 199.

Hengstler, M., Enkel, E. and Duelli, S. (2016), "Applied artificial intelligence and trust-the case of autonomous vehicles and medical assistance devices", Technological Forecasting and Social Change, Vol. 105, pp. 105-120.

Ivanco, A. (2017), "Fleet analysis of headway distance for autonomous driving", Journal of Safety Research, Vol. 63, pp. 145-148.

Jung, C., Lee, D., Lee, S. and Shim, D.H. (2020), "V2X-Communication-Aided autonomous driving: system design and experimental validation", Sensors, Vol. 20 No. 10, p. 2903.

Leek, M.R. (2001), "Adaptive procedures in psychophysical research", Perception and Psychophysics, Vol. 63 No. 8, pp. 1279-1292, doi: 10.3758/BF03194543. 
FEBE 1,2

Lewis-Evans, B., De Waard, D. and Brookhuis, K.A. (2010), “That's close enough-a threshold effect of time headway on the experience of risk, task difficulty, effort, and comfort", Accident Analysis and Prevention, Vol. 42 No. 6, pp. 1926-1933.

Li, Y., Chen, S., Dong, J., Steinfeld, A. and Labi, S. (2020a), "Leveraging vehicle connectivity and autonomy to stabilize flow in mixed traffic conditions: accounting for human-driven vehicle driver behavioral heterogeneity and perception-reaction time delay", arXiv:2008.04351, available at: https://arxiv.org/abs/2008.04351.

Li, Y., Chen, S., Du, R., Ha, P., Dong, J. and Labi, S. (2020b), "Using empirical trajectory data to design connected autonomous vehicle controllers for traffic stabilization”, arXiv preprint arXiv: 2010.05440, available at: https://arxiv.org/abs/2010.05440.

Martin-Gasulla, M., Sukennik, P. and Lohmiller, J. (2019), "Investigation of the impact on throughput of connected autonomous vehicles with headway based on the leading vehicle type", Transportation Research Record, Vol. 2673 No. 5, pp. 617-626.

Merat, N., Jamson, A.H., Lai, F.C., Daly, M. and Carsten, O.M. (2014), "Transition to manual: driver behaviour when resuming control from a highly automated vehicle", Transportation Research Part F: Traffic Psychology and Behaviour, Vol. 27, pp. 274-282.

Miller, D., Johns, M., Mok, B., Gowda, N., Sirkin, D., Lee, K. and Ju, W. (2016), "Behavioral measurement of trust in automation: the trust fall", Proceedings of the Human Factors and Ergonomics Society Annual Meeting, Vol. 60 No. 1, pp. 1849-1853, Sage CA, SAGE Publications, Los Angeles, CA.

Molnar, L.J., Ryan, L.H., Pradhan, A.K., Eby, D.W., Louis, R.M. and Zakrajsek, J.S. (2018), "Understanding trust and acceptance of automated vehicles: an exploratory simulator study of transfer of control between automated and manual driving", Transportation Research Part F: Traffic Psychology and Behaviour, Vol. 58, pp. 319-328.

Ni, R., Kang, J.J. and Andersen, G.J. (2010), "Age-related declines in car following performance under simulated fog conditions", Accident Analysis and Prevention, Vol. 42 No. 3, pp. 818-826.

Patten, C.J., Kircher, A., Östlund, J., Nilsson, L. and Svenson, O. (2006), "Driver experience and cognitive workload in different traffic environments", Accident Analysis and Prevention, Vol. 38 No. 5, pp. 887-894.

Payre, W., Cestac, J. and Delhomme, P. (2016), "Fully automated driving: impact of trust and practice on manual control recovery", Human Factors, Vol. 58 No. 2, pp. 229-241.

Petersen, L., Robert, L., Yang, J. and Tilbury, D. (2019), "Situational awareness, driver's trust in automated driving systems and secondary task performance", SAE International Journal of Connected and Autonomous Vehicles, SAE, Vol. 2 No. 2, pp. 129-141, doi: 10.4271/12-02-02-0009.

Piccinini, G.F.B., Rodrigues, C.M., Leitão, M. and Simões, A. (2014), "Driver's behavioral adaptation to adaptive cruise control (ACC): the case of speed and time headway", Journal of Safety Research, Vol. 49, pp. 77-84.

Risto, M. and Martens, M.H. (2013), "Time and space: the difference between following time headway and distance headway instructions", Transportation Research Part F: Traffic Psychology and Behaviour, Vol. 17, pp. 45-51.

SAE International (2018), "Taxonomy and definitions for terms related to driving automation systems for on-road motor vehicles", SAE International, available at: https:/www.sae.org/standards/ content/j3016_201806/, doi: 10.4271/J3016_201806.

Saifuzzaman, M., Haque, M.M., Zheng, Z. and Washington, S. (2015), "Impact of mobile phone use on car-following behaviour of young drivers", Accident Analysis and Prevention, Vol. 82, pp. 10-19.

Shinar, D. and Schechtman, E. (2002), "Headway feedback improves intervehicular distance: a field study", Human Factors, Vol. 44 No. 3, pp. 474-481.

Shinar, D., Tractinsky, N. and Compton, R. (2005), "Effects of practice, age, and task demands, on interference from a phone task while driving", Accident Analysis and Prevention, Vol. 37 No. 2, pp. 315-326. 
Siebert, F.W., Oehl, M. and Pfister, H.R. (2014), "The influence of time headway on subjective driver states in adaptive cruise control", Transportation Research Part F: Traffic Psychology and Behaviour, Vol. 25, pp. 65-73.

Simpson, W.A. (1988), "The method of constant stimuli is efficient", Perception and Psychophysics, Vol. 44 No. 5, pp. 433-436, doi: 10.3758/BF03210427.

Comfortable vs mobilityenhancing headways

Suzuki, H. and Nakatsuji, T. (2015), "Dynamic estimation of headway distance in vehicle platoon system under unexpected car-following situations", Transportation Research Procedia, Vol. 6, pp. 172-188, doi: 10.1016/j.trpro.2015.03.014.

Taieb-Maimon, M. and Shinar, D. (2001), "Minimum and comfortable driving headways: reality versus perception”, Human Factors, Vol. 43 No. 1, pp. 159-172, doi: 10.1518/001872001775992543.

Underwood, G. (2013), "On-road behaviour of younger and older novices during the first six months of driving", Accident Analysis and Prevention, Vol. 58, pp. 235-243.

Vob, G.M., Keck, C.M. and Schwalm, M. (2018), "Investigation of drivers' thresholds of a subjectively accepted driving performance with a focus on automated driving", Transportation Research Part F: Traffic Psychology and Behaviour, Vol. 56, pp. 280-292.

Vogel, K. (2003), "A comparison of headway and time to collision as safety indicators", Accident Analysis and Prevention, Vol. 35 No. 3, pp. 427-433.

Winsum, W.V. and Heino, A. (1996), "Choice of time-headway in car-following and the role of time-tocollision information in braking", Ergonomics, Vol. 39 No. 4, pp. 579-592.

\section{About the authors}

Yujie Li is a graduate student of Lyles School of Civil Engineering at Purdue University and research staff at the Center for Connected and Automated Transportation (CCAT). She received her BS in Transportation Engineering from Southeast University and MS in civil engineering from Purdue University. Her current research interests include connected and autonomous vehicle control and human factors associated with emerging AV technologies.

Tiantian Chen is a postdoctoral fellow in the Department of Industrial and Systems Engineering at The Hong Kong Polytechnic University. Her research interests include safety evaluation for professional drivers, driving simulator studies, driver behavior, and transport policy. Her awards include the Best Paper Award in the 5th International Conference on Transportation Information and Safety (2019) in Liverpool, United Kingdom, the HKSAR Government Scholarship Fund for attending the 6th International Choice Modelling Conference (2019) in Kobe, Japan. She is also the winner of the OSH Best Project Scholarship 2019/2020 awarded by the Hong Kong Occupational Safety and Health Council.

Sikai Chen is a Visiting Assistant Professor at the Center for Connected and Automated Transportation (CCAT) and Lyles School of Civil Engineering at Purdue University, and Visiting Research Fellow at the Robotics Institute, School of Computer Science at Carnegie Mellon University. His research interests span both theoretical and applied aspects of artificial intelligence, data science, robotics, and optimization for autonomous vehicle operations and transportation and infrastructure systems. Dr. Chen serves as co-PI for USDOT-funded research projects at CCAT. In addition, he is a member of two ASCE national committees: Connected and Autonomous Vehicle Impacts, and Economics and Finance. Sikai Chen is the corresponding author and can be contacted at: chen1670@purdue.edu

Samuel Labi is a professor of civil engineering at Purdue University and associate director of USDOT's Center for Connected and Automated Transportation. He chairs the American Society of Civil Engineers (ASCE)'s Economics and Finance committee and is associate editor of Computer-Aided Civil and Infrastructure Engineering and ASCE Journals of Risk and Uncertainty, and Infrastructure Systems. Dr. Labi is author of Introduction to Civil Engineering Systems (Wiley) and co-author of Transportation Decision-Making (Wiley). His awards include TRB's Mickle Award in 2019 for best paper in maintenance and operations and K.B. Woods Prize in 2008 for outstanding paper in design and construction.

For instructions on how to order reprints of this article, please visit our website:

www.emeraldgrouppublishing.com/licensing/reprints.htm

Or contact us for further details: permissions@emeraldinsight.com 